\title{
USP13 Gene
}

National Cancer Institute

\section{Source}

National Cancer Institute. USP13 Gene. NCI Thesaurus. Code C115004.

This gene is involved in promoting protein stability. 